\title{
Vinos típicos de Chile: ascenso y declinación del chacolí (1810-2015)
}

\author{
Typical Wines of Chile: the rise and decline of Chacoli (1810-2015) \\ Pablo Lacoste $^{1 *}$, Amalia Castro ${ }^{2}$, Félix Briones $^{3}$, Felipe Cussen $^{1}$, \\ Natalia Soto ${ }^{4}$, Bibiana Rendón ${ }^{5}$, Fernando Mujica ${ }^{6}$, Paulette Aguilera ${ }^{1}$, Carolina Cofré ${ }^{1}$, \\ Emiliano Núñez ${ }^{1}$, Michelle L. Adunka ${ }^{1}$
}

\begin{abstract}
RESUMEN
Este artículo examina el surgimiento, consolidación y decadencia del chacolí, vino típico chileno elaborado en el siglo XIX y parte del XX. Heredero de la tradición española colonial, el chacolí chileno se elaboraba a partir de Uva País, Moscatel de Alejandría y variedades criollas, sobre todo Torontel. En el último tercio del siglo XIX llegó a representar el 28\% de la producción chilena de vinos. Con posterioridad comenzó a decaer, muy criticado por los tecnócratas europeos y sus seguidores chilenos, que negaron todo valor enológico a las variedades utilizadas y los métodos artesanales. De todos modos, el chacolí se ha mantenido latente en pequeños segmentos de consumidores, sobre todo de segmentos populares y campesinos; además, desde 1975 se reivindica en la Fiesta del Chacolí, celebrada anualmente en Doñihue, en el corazón profundo de la huasa provincia de Colchagua.
\end{abstract}

Palabras clave: Chacolí chileno, vinos típicos, vitivinicultura chilena.

\begin{abstract}
This article examines the emergence, consolidation and decline of Chacolí, typical Chilean wine. Heir to the Spanish colonial tradition, the Chilean Chacoli was made from Uva País (called Mission in California), Muscat of Alexandria and landraces, especially Torontel (Yellow Muscat). In the last third of the nineteenth century, Chacoli represented 28\% of Chilean wine production. This typical wine then began to decline, strongly criticized by European technocrats and their Chilean fans who denied any value wine varieties used and artisanal methods. Anyway, the Chacoli has remained latent in small segments of consumers, especially popular segments and peasants; moreover, since 1975 it claimed in Chacoli Festival, held annually in Doñihue, in the deep heart of the traditional province of Colchagua.
\end{abstract}

Key words: Chilean Chacolí, typical wines, Chilean wine.

\section{Introducción}

El chacolí es un vino típico de la vitivinicultura popular de Chile, elaborado desde el Huasco, en el Norte Chico, hasta el Valle Central. Se trata de una bebida de singular tradición, ampliamente difundida en las masas populares, sobre todo en las celebraciones de fin de cosecha, carnaval y fiestas patrias. Para muchos chilenos, esta bebida es parte importante de su vida social y de su identidad cultural.
La gran industria vitivinícola chilena ha dedicado escaso interés al desarrollo de estas bebidas. Ha priorizado el enfoque angloamericano de los puntos Parker y la estandarización de los productos. No se ha preocupado por la identidad territorial ni histórica de los vinos. Esta situación ha adquirido relevancia por la fuerte concentración de la industria en las grandes empresas. Basta recordar que la mayor de ellas, Concha y Toro, controla el 25\% del mercado nacional; y las diez mayores manejan el $50 \%$ de las exportaciones. Para aprovechar su

\footnotetext{
Universidad de Santiago de Chile, USACH. Santiago, Chile. Universidad Finis Terrae. Santiago, Chile.

Universidad del Bío Bío. Chillán, Chile.

Universidad Nacional de Cuyo. Mendoza, Argentina.

Universidad de Chile. Santiago, Chile.

6 Escuela Nacional de Sommelier. Santiago, Chile.

* Autor por correspondencia: pablo.lacoste@usach.cl
}

Fecha de Recepción: 14 Enero, 2015.

Fecha de Aceptación: 15 Mayo, 2015. 
posición dominante, estas grandes empresas han optado por promover los vinos estandarizados, sin interesarse por las denominaciones de origen y los productos típicos.

Esta bebida típica no ha sido estudiada hasta el momento con suficiente profundidad por la académica. Los trabajos de Claudio Gay (1855) trazaron un perfil un tanto despectivo de este producto y sentaron las bases de su valoración negativa, reiterada después por los autores posteriores (Couyoumdjian, 2006; Del Pozo, 2014). Algunos trabajos dedicados a la historia del vino chileno se han focalizado principalmente en las grandes viñas (Del Pozo, 2014), el problema del alcoholismo (Fernández Labbé, 2010) y el proceso de modernización impulsado por los franceses en la segunda mitad del siglo XIX (Briones, 2006; Coujoumdjian, 2006), el terroir y las cepas francesas (Pszczólkowski, 2014 y 2015). Naturalmente, estos autores se focalizaron en la corriente principal de la industria vitivinícola chilena, dejando para más adelante las ramas secundarias, dentro de las que se encuentran, precisamente, el chacolí.

Las referencias parciales de la literatura especializada en la historia del vino chileno trazan algunos antecedentes para conocer estos productos. Pero han dejado muchas preguntas abiertas. Por un lado, conviene identificar qué uvas se utilizaban para elaborar el chacolí. Gay (1855) y Del Pozo (2014) lo asocian con las uvas criollas, pero conviene definir con mayor claridad el concepto. También es importante determinar el área geográfica del chacolí, considerando también los estudios de Morales (1896) para la zona del Huasco. Otro elemento que conviene aclarar es el ciclo histórico de estos productos. Uno de los estudios entrega datos de la rentabilidad económica del chacolí por hectárea de viña en 1874 en Los Andes y San Felipe (Aránguiz, 1995). Queda pendiente sistematizar la información disponible, enriquecerla con nuevas fuentes y establecer con mayor precisión la historia, identidad y ubicación geográfica de esta bebida, objetos del presente artículo.

Para alcanzar estos objetivos, el presente artículo ha examinado cuatro corpus documentales principales: a) inventarios de bienes y testamentos de los viticultores chilenos de la primera mitad del siglo XIX, conservados en el Archivo Nacional (AN); b) Fondos de Tesorería y Aduana de Chile en el segundo cuarto del siglo XIX (AN); c) medios de prensa gráfica editados en Santiago, Valparaíso y otras ciudades chilenas, entre la segunda mitad del siglo XIX y el primer tercio del XX; y d) Archivo del Instituto Nacional de Propiedad Industrial (INAPI). Se ha utilizado el método propio de la historia (heurístico crítico) para confrontar las hipótesis con los referentes empíricos mencionados. Todo ello se ha interpretado a la luz de la bibliografía especializada sobre el tema. Como resultado, se ha podido reconstruir el itinerario cultural de este producto en Chile.

\section{Del chacolí vasco al chileno}

El chacolí chileno tiene un sinónimo en el chacolí español. En realidad, se trata de dos vinos diferentes con el mismo nombre. La homonimia se produjo debido a un largo proceso cultural signado por la inmigración de vascos a Chile, quienes viajaron acompañados por sus usos, costumbres y tradiciones, entre las que se encontraba el chacolí. Una vez arraigados en Chile, los inmigrantes vascos trataron de recrear las condiciones de vida de su país de origen $y$, entre otros productos, impulsaron la elaboración del chacolí. Pero era un nuevo producto, resultado de la interacción entre las tradiciones españolas y las realidades del nuevo mundo.

En España se conoce como "Chacolí al vino de poco cuerpo, ácido y de baja graduación y con cierta agujilla de carbónico, producido en los tres territorios del País Vasco, zonas de Cantabria y mitad septentrional de la provincia de Burgos, lugares todos ellos donde la uva no alcanzaba una maduración completa. El Chacolí es un vino ligero, ácido y afrutado de poco grado, se ha producido desde hace más de mil años en los territorios vascos y zonas de Burgos y Cantabria. El factor común de los caldos de los distintos territorios ha sido la falta de madurez adecuada de la uva" (Hidalgo, 2012).

En cambio el chacolí chileno es un producto algo distinto. Se elabora a partir de uvas maduras de cepa país, es un vino joven, sin envejecimiento en barrica ni guarda en botella. La valiosísima Cartilla de campo y otras curiosidades, dirigidas a la enseñanza y buen éxito de un hijo, interesante instructivo que comprende los años 1808 a 1817 y en el que se educa sobre las costumbres y labores del campo chileno, hace mención a este brebaje precisando que: "Es el caldo que la uva produce en la primera pisa, en la segunda, y de la estruja que se le hace con la tabla en un lado del lagar" (Fernández 
Niño, 1817). En su segunda edición anuncia de modo más claro: "Mosto Chacolí. Es el caldo que la uva produce en la primera y segunda pisa, y el de la estruja que vierte la tabla antes de levantarse el orujo al suncho" (Fernández Niño, 1867).

De todos modos existe una diferencia importante entre los dos productos: los vascos han puesto en valor su chacolí con el desarrollo de tres Denominaciones de Origen: Chacolí de Getaria-GetariakoTxakolina (1989); Chacolí de Bizkaia-BizkaikoTxakolina (1994) y Chacolí de Ávala-ArabakoTxacolina (2001) (Hidalgo, et. al. 2012). En cambio en Chile, el chacolí ha quedado marginado como un vino de tercera o cuarta clase.

Pero no siempre fue así. El capitán inglés Ricardo Longueville Vowell, quien se encontraba al servicio de Chile entre 1821 y 1829 , en su recorrido por el territorio apreció favorablemente los subproductos de las viñas, señalando que: "En Chile las viñas son abundantes y de sus uvas se hace muy buen vino y aguardiente. Se fabrican otras bebidas como el Chacolí y el sancochado" (Hanisch, 1976). En el exquisito detalle que brinda de una cena a la que fue invitado en Tabolango, comuna de Quillota, el chacolí también se hace presente: "Grandes cachos de chacolí y vino circulaban de mano en mano en rápida sucesión" (Hanisch, 1976). Otro viajero, esta vez francés, el capitán Lafond, explica otro de los usos de este brebaje: "En la tarde se come arroz con leche y galletas y como postre las frutas de la estación, melones, brevas, fresas. Todo regado con abundante chacolî" (Pereira, 2013).

Mayor espacio en sus escritos le brindó Claudio Gay (1855), el cual definió el chacolí en los siguientes términos: "es el mosto fermentado que no contiene arrope ni cocido. En algunos puntos se le prepara pisando las uvas sobre una estera y en seguida se hace fermentar el jugo en una tinaja, pudiéndose beberle al cabo de seis días. Privada como se ve de un suplemento dulce no puede conservarse mucho tiempo, acaso porque los riegos extremadamente fecundos en Chile dan una fuerza tal que hace que las uvas contengan demasiadas sustancias azotadas para ser enteramente absorbidas por la fermentación, y este exceso es lo que la deteriora al cabo de cinco a seis meses. También la falta de tanino ocasiona este daño y en este caso se la haría más duradera si el racimo con su escobajo se pusiese a fermentar. Esta bebida es delgada, suave y de un sabor agridulce que la asemeja a la sidra. Su consumo es muy grande, sobre todo, poco después de la vendimia" (Gay, 1855).

En el ciclo de auge de los productos típicos del campo chileno, algunos viticultores se especializaron en elaborar este tipo de vino. Los documentos han permitido detectar evidencia sobre algunos productores y comerciantes relevantes dedicados al chacolí.

El Norte Chico fue uno de los principales centros de producción de chacolí. El registro más antiguo corresponde a Antonio Zavala, quien hacia 1810 elaboraba 100 @ de chacolí en el Huasco. Poco después, en 1824, se detectó que en Vallenar se elaboraban entre 2.000 y 3.000 @ de vino, entre mosto y chacolí (Morales, 1896). Posteriormente, en 1832 se levantó un nuevo registro, el que permitió comprender la relevancia que el chacolí había alcanzado en el valle del Huasco (Tabla 1).

El chacolí también se elaboró con fines comerciales en la zona central de Chile desde las primeras décadas del siglo XIX. Entre los registros más antiguos figuran Joseph Galdame (1834), Francisco Prats (1837), Alejo Lemus (1841-1844), José Dolores Chacón (1845), Ceciliano Álvarez (1845) y Pedro Figueroa (1844).

Hacia 1837 don Francisco Prats, propietario de una viña de 11.000 plantas, tenía en su bodega 471,5 @ de chacolí, valuado a siete reales cada arroba. ${ }^{1}$

Tabla 1. Productores de chacolí en el valle del Huasco (1824-1832)

\begin{tabular}{lcc}
\hline Nombre del productor & Nombre de la Hacienda & Producción \\
\hline Nicolás Urquieta & Chacra la Peona & 800 parras-50 @ de chacolí \\
Mateo Páez & Chacra de Mateo Páez & $40 @$ de chacolí \\
José María Rodríguez & Chacra (hoy) de Araya & 5.500 parras-200 @ de chacolí \\
Nicolás Ahumada & Chacra de Nicolás Ahumada & 700 parras-50 @ de chacolí \\
Juan Pérez & Chacra de Juan Pérez & 500 parras-26 @ de chacolí \\
José Antonio Zavala & Chacra (hoy) de los Alemanes & 6.000 parras-200 @ de chacolí \\
\hline
\end{tabular}

Fuente: elaboración propia a partir de los datos entregados por Joaquín Morales sobre el informe de 1824 y el informe estadístico de 1832 (Morales, 1981: 221-226). 
La chacra de Miraflores (Ñuñoa), propiedad de don Alejo Lemus, fue otro centro productivo de chacolí. Entre 1841 y 1844 esta propiedad fue arrendada y se generó un pleito, gracias al cual se registró la producción de la hacienda. Los documentos señalan que "En el año de 1842 (se elaboraron) 73 arrobas de chacolí a 12 reales arroba: \$ 109 con 6 reales; en el año de 1843 del chacolí que se vendió le entregué 461 pesos, cuyo precio fue el de 10 reales arroba: \$ 461". ${ }^{2}$ En este segundo año, la producción llegó a370@ de chacolí.

Una década más tarde se produjo el registro de la operación de Chacón. El 11 de julio de 1845 en Santiago de Chile, don José vendió a don Ceciliano Álvarez tres cubas de chacolí de 100 @, por valor de $\$ 157$ con cuatro reales. Poco después, don Ceciliano se declaró en quiebra, sin haber alcanzado a pagar el precio convenido. Por lo tanto, don José solicitó la anulación de la venta y la recuperación de su chacolí. ${ }^{3}$ Mientras tanto, para hacer frente a sus acreedores, Álvarez propició un inventario completo de sus bienes, entre los que había mil @ de chacolí, tasadas a siete reales por arroba, totalizando un valor de $\$ 1500 .^{4}$

Fuera de Santiago, 100 kilómetros al nordeste de la capital, en San Felipe, también se documentó la presencia del chacolí. En la hacienda de Joseph Galdame se inventariaron 20 @ de chacolí, valuadas a 12 reales por arroba, con un total de $\$ 30 .{ }^{5}$ Entre los bienes de Pedro Figueroa se registraron 16 @ de chacolí, valuadas a ocho reales por arroba. ${ }^{6}$ A mediados de la década de 1850, Claudio Gay ponderó que la arroba de chacolí valía ocho reales en 1841 (Gay, 1855).

La extensión de la navegación de cabotaje, a mediados del siglo XIX, facilitó la distribución del chacolí por todo el país. En 1842 el bergantín Castro zarpó de Coquimbo, con escala en Valparaíso y destino final en Chiloé, donde llegó con "dos barrilitos de chacolí moscatel" (Ankud, 31 de diciembre de 1842, Fondo Tesorería y Aduana de Chiloé, vol 50, comprobantes 1842 p. 21). En 1849 llegaron a ese puerto una pipa de chacolí procedente de Talcahuano y tres barriles de chacolí de Coquimbo (Intendencia Chiloé, Fondo Ministerio del Interior, vol 251 p. 536).

A medida que la expansión del transporte alentaba el comercio, los productores se animaron a fortalecer la oferta de chacolí. Uno de los centros productivos más interesantes fue el fundo San Pedro, pequeño viñedo de dos hectáreas dedicado exclusivamente a elaborar y comercializar chacolí. La viña tenía atributos para entroncarse dentro de la viticultura tradicional de Chile. Estaba cercada por muros de tapia, de dos metros de altura. Solo se cultivaba cepa de Uva País. El método de cultivo era el de cabeza doble (dos parras juntas), con parras altas (1,40 metros). El único producto que se fabricaba era el chacolí y como producto accesorio se destilaba el orujo para sacar aguardiente. Junto a la viña se hallaban los edificios de vivienda y bodega, donde se encontraban las cubas de fermentación, con25@de capacidad, y los toneles, con60@ de capacidad.

La vendimia del "Fundo San Pedro" se realizaba en abril y duraba de tres a seis días. Participaban diez a doce mujeres, lideradas por el mayordomo. La uva se cortaba con cuchillo y se recogía en canastos de mimbre de 20 litros de capacidad. Una vez en la bodega, la uva se presionaba sobre una estera de coligüies colocada sobre las cubas fermentadoras. Esta operación la ejecutaban con la mano dos individuos colocados uno en frente del otro. Cuando se llenaba una cuba se trasladaba el aparato a otra y se repetía ahí la operación.

La elaboración del vino se realizaba en la artesanal bodega. 48 horas después de la vendimia comenzaba la fermentación y se prolongaba hasta cuarto o quinto día. Posteriormente el vino se retiraba de las cubas fermentadoras para pasarlo a los toneles, donde permanecía durante un mes. Cumplido este plazo, el vino se trasegaba y ya se lo consideraba producto terminado. De este modo se obtenía el chacolí, que se vendía a 2 pesos la arroba en la misma bodega. Los toneles se rellenaban cada 15 días. Las tapas se ajustaban bien y se les ponía una mezcla de ceniza y agua que, al secarse, impedía la entrada de aire (Alessandri, 1885).

El caso del "Fundo San Pedro" tiene singular interés para comprender el significado social, económico y cultural del chacolí en el Valle Central de Chile. Era un producto artesanal, elaborado con métodos tradicionales, sin incorporación de productos químicos ni aditivos para "corregir" el color, aroma y sabor. No se elaboraba en un gran establecimiento industrial, como se estilaba en el modelo de grandes fábricas de vino que se estaba asentando en esa época en el Cono Sur de América. Tampoco requería instalaciones ni equipamientos sofisticados traídos de Francia, como las moledoras mecánicas de uva o los grandes toneles de roble. Además, el chacolí se vendía al público en la 
misma bodega, sin depender de las grandes cadenas comercializadoras. Era un paradigma de pequeños viticultores que cultivaban la viña y elaboraban sus propios vinos.

\section{La edad dorada del chacolí en Chile}

El siglo XIX fue la edad dorada del chacolí en Chile. Justo antes del proceso de afrancesamiento de la viticultura chilena, este producto alcanzó su ciclo de apogeo. Mientras los enólogos y viticultores de las nuevas tendencias se empeñaban en incorporar las uvas francesas en Chile para elaborar los "vinos tipo burdeos", la viticultura tradicional siguió elaborando chacolí con los métodos de siempre, hasta alcanzar volúmenes importantes.

El chacolí ocupó un lugar relevante en la industria vitivinícola nacional en el último tercio del siglo XIX. Fue un producto central de la agroindustria nacional. El Estado ordenó levantar datos permanentemente sobre las cantidades de producción. El análisis de estos relevamientos muestra que los viticultores chilenos dedicaron una parte sustancial de sus energías productivas al chacolí. En efecto, su producción anual llegaba muy cerca de la producción de vinos y mostos. Así se desprende de los Anuarios del Instituto Nacional de Estadística (INE), entre 1861 y 1890 (Tabla 2).

La visión de conjunto de estas tablas permite comprender la importancia relativa del chacolí dentro del escenario general de la industria vitivinícola chilena. En las tres décadas examinadas, sobre un total de 1.724 millones de litros de vinos y chichas, se elaboraron 487 millones de litros de chacolí, lo que representa el $28,2 \%$ del total. En otras palabras, el chacolí comprendía casi un tercio de la industria vitivinícola de Chile.

Tabla 2. Producción vitivinícola de Chile (1861-1890) (en litros).

\begin{tabular}{|c|c|c|c|}
\hline Año & Chacolí & Chicha & Vino y mosto \\
\hline 1861 & 10.121 .938 & 18.189 .127 & 25.015 .786 \\
\hline 1862 & 15.185 .622 & 20.972 .512 & 26.232 .228 \\
\hline 1863 & 12.719 .615 & 24.846 .595 & 21.741 .585 \\
\hline 1864 & 15.830 .941 & 27.062 .207 & 26.912 .444 \\
\hline 1865 & - & - & - \\
\hline 1866 & 15.830 .941 & 22.793 .839 & 25.329 .676 \\
\hline 1867 & 16.353 .643 & 23.684 .359 & 28.640 .584 \\
\hline 1868 & 16.232 .839 & 24.253 .564 & 23.501 .926 \\
\hline 1869 & 18.012 .878 & 21.217 .071 & 23.129 .635 \\
\hline 1870 & 14.449 .285 & 23.311 .897 & 20.582 .015 \\
\hline 1871 & 14.192 .659 & 23.311 .897 & 25.974 .429 \\
\hline 1872 & 20.685 .744 & 20.140 .853 & 31.327 .579 \\
\hline 1873 & 21.458 .295 & 21.279 .906 & 32.131 .689 \\
\hline 1874 & 19.668 .207 & 20.702 .961 & - \\
\hline 1875 & 21.552 .121 & 20.157 .148 & 29.658 .805 \\
\hline 1876 & 20.044 .152 & 25.812 .588 & 26.511 .720 \\
\hline 1877 & 20.024 .726 & 20.391 .191 & 20.507 .325 \\
\hline 1878 & 18.340 .156 & 17.780 .576 & 24.701 .685 \\
\hline 1879 & 19.319 .070 & 18.206 .165 & 21.796 .483 \\
\hline 1880 & 28.059 .418 & 20.215 .595 & 19.454 .716 \\
\hline 1881 & - & - & - \\
\hline 1882 & 17.680 .558 & 19.568 .142 & 28.659 .515 \\
\hline 1883 & 22.825 .284 & 21.994 .551 & 38.780 .905 \\
\hline 1884 & 19.675 .158 & 22.800 .150 & 27.628 .947 \\
\hline 1885 & 17.449 .366 & 18.490 .473 & 28.949 .084 \\
\hline 1886 & 15.239 .653 & 17.529 .216 & 27.636 .753 \\
\hline 1887 & 12.292 .129 & 12.992 .462 & 20.843 .680 \\
\hline 1888 & 11.152 .403 & 12.036 .735 & 21.236 .214 \\
\hline 1889 & 5.802 .434 & 7.148 .815 & 12.375 .129 \\
\hline 1890 & 9.349 .345 & 10.471 .621 & 17.579 .835 \\
\hline Total & 487.229 .137 & 561.362 .216 & 676.840 .172 \\
\hline
\end{tabular}

Fuente: Anuario Estadístico de la República de Chile, 1861-1890. 
El chacolí se destacaba no solo por el volumen de producción, sino también por su voluntad de alcanzar mejores niveles de calidad, presentarse y competir en las exposiciones de la época. En efecto, en la segunda mitad del siglo XIX, a pesar del desprecio generado por las élites intelectuales y los tecnócratas, los productores de estos vinos típicos lograban destacarse en las competencias. Por ejemplo, en la Exposición Departamental de Los Andes, los chacolíes tuvieron trece exponentes, obteniendo un segundo premio y tres menciones honrosas (Le Feuvre et al., 1886).

El chacolí se dividía en dos clases: blanco y morado, que luego derivó en rosado. A mediados del siglo XIX, Claudio Gay explicaba la diferencia en los siguientes términos: "Hay de dos clase, una blanca, fermentada sin hollejos, y la otra morada por haberse mezclado estos hollejos y escobajos en gran abundancia al tiempo de la fermentación" (Gay, 1855). Décadas más tarde se produjo un refinamiento. Una valoración especial merecía el chacolí blanco elaborado con uvas torontel ( $E l$ Mercurio, 9 de octubre de 1915; 16 de octubre de 1915). También se ofrecía el "chacolí Morado de Superior Clase" (El Comercio de San Felipe, 20 de agosto de 1888) en el aviso de la Hacienda San José. Posteriormente, en el siglo XX, se comenzó a usar la expresión chacolí rosado. La bodega del Patriarca Noé, ya mencionada, promovía el chacolí rosado. Más adelante, otro aviso promovía el "chacolí Rosado de Molina”, en venta en una casa de comercio ubicada en calle Riquelme $\mathrm{N}^{\circ} 258$, entre las calles Huérfanos y Agustinas, en pleno centro de Santiago (El Mercurio, 3 de noviembre de 1914).

La importancia del consumo de estos productos queda de manifiesto en los avisos comerciales publicados en la segunda mitad del siglo XIX y en el primer tercio del XX. Para aquellos que "necesitaban de un chacolí bueno, podían con confianza dirigirse a la calle San Isidro de la Cañada segunda cuadra, en la Barraca de Madera", y allí lo hallarían en venta.

Los pequeños viticultores al promover sus productos típicos reflejaban la actitud de orgullo que sentían como vignerons, como artífices de sus propios vinos. Ellos asociaban sus vinos con la calidad de vida, se esforzaban por elaborar de la mejor manera posible sus vinos, se comprometían con sus trabajos y luego los promocionaban desde el orgullo y la satisfacción de haberlo hecho bien. Esa convicción los estimulaba para elaborar, promover y comercializar sus propios vinos.

\section{Los tecnócratas europeos y el desprecio de los productos típicos chilenos}

Los tecnócratas europeos lideraron la transformación de la viticultura chilena en la segunda mitad del siglo XIX. Revestidos del prestigio que irradiaban sus países de origen, lograron posicionarse en el centro de la formación de opinión sobre la valoración de los productos del campo y la jerarquía de los alimentos en todo el país. Ellos realizaron sus acciones en un ambiente sociocultural de admiración ilimitada de las élites chilenas por la cultura europea en general y francesa en particular, proceso que fue vivido también en el resto de América Latina. En este contexto, ellos gozaron de un poder y una influencia decisivos en el proceso de modelar los gustos y tendencias. Tanto Claudio Gay (1865) como René Le Feuvre (1877 y 1890), Julio Menadier (1874), Riveros (1881) y Charlin (1881) participaron de esta corriente. Ellos dirigieron institutos de formación técnica y publicaciones especializadas desde donde marcaron una línea de pensamiento que se convirtió en hegemónica. Las élites adoptaron los criterios propuestos por estos tecnócratas, lo que se hizo notar en la vida económica, comercial y cultural, particularmente en las pautas de consumo de alimentos y bebidas.

En el plano de la vitivinicultura, los tecnócratas europeos instalaron dos tendencias: por un lado, la sobrevaloración de las cepas francesas y los vinos imitación, tipo burdeos, borgoña, champagne, etcétera. Por otra parte, esta tendencia se vio acompañada con una actitud de desprecio hacia las variedades de uva criollas (Uva País, Moscatel de Alejandría, Moscatel Amarillo, Torrontés, Moscatel de Austria, entre otros). Para aquellos tecnócratas esas variedades no tenían valor enológico y no era posible elaborar con ellas vinos de buena calidad. Instalaron una cultura de la minimización del valor de las variedades criollas, concepto que se transformó en paradigma hegemónico en las ciencias agrarias chilenas y mantuvo esta posición durante más de un siglo. Recién se comenzaría a revertir a comienzos del siglo XXI, con exitosos ensayos de vinos, espumantes y piscos de Uva País y Moscatel de Alejandría.

Además de despreciar las variedades criollas, los tecnócratas europeos del siglo XIX despreciaron la viticultura tradicional en su conjunto. En lugar de valorar la diversidad de métodos y estilos, propiciaron que solo fuera aceptable la aplicación 
de los sistemas franceses. Negaron el valor de las viñas de rulo y las cepas de cabeza. También censuraron el equipamiento y las instalaciones de los viticultores artesanales, como lagares de cuero y pipas de roble chileno. Finalmente, negaron todo valor a los productos típicos como el chacolí. En líneas generales, para estos autores estos productos eran de mala calidad por provenir de viñas mal cultivadas y usar deficientes métodos de elaboración, particularmente el chacolí (Le Feuvre, 1884). Los textos dedicados a analizar la situación chilena del agro y recomendar cambios para el "mejoramiento" de la industria insistían en cuestionar estos productos:

"Los productos más comunes de nuestros viñedos: los chacolíes son de tan imperfecta elaboración que por su mala calidad y aun por su insalubridad debieran proscribirse en lugar de permitir que cada año se aumente su producción y consumo" (Menadier, 1874).

El repetido martilleo de los tecnócratas extranjeros se extendió también a los enólogos y agrónomos chilenos. Los manuales de estudio y los centros de enseñanza, al tratar los temas de viticultura y vinificación, terminaron por consolidar el paradigma afrancesado propuesto. Como resultado, en el siglo XX se impuso en la viticultura chilena una tendencia a reproducir el modelo de las viñas centradas en variedades francesas, con métodos franceses, adaptados al estilo de las grandes fábricas de vino, en manos de un reducido número de familias ricas.

Hasta fines del siglo XIX, la producción de los vinos típicos seguía siendo importante, pero a partir de entonces comenzó a perder terreno frente al avance de los vinos al estilo francés. "En 1883 se producían en Chile 41,7 millones de litros de vino contra 31 millones de chacolí; en 1923, las cifras eran de 243 millones de litros de vino contra 57 millones para el chacolí, respectivamente" (Del Pozo, 1998).

Las décadas de 1850, 1860 y 1870 fueron una fase de transición, en la que el vino país, así como el chacolí y el aguardiente eran expuestos en el mismo escenario con el vino "de imitación francesa" que estaba lejos de predominar. En efecto, según cifras de 1869 , la casi totalidad del vino producido en Chile era el del primer tipo que alcanzaba a651.000@ o 25 millones de litros, de los que casi la mitad se producían en Concepción, con 250.000 @, seguida de Maule con113.000 @; Santiago solo rendía 19.000 @. Del "vino Burdeos", del que se hacía contabilidad aparte, se produjeron 22.800 @, casi todas en Santiago. La producción de chacolí seguía siendo muy importante, con cifras de 507.000 @, la mitad de lo que se elaboraba en Santiago (Del Pozo, 1998).

\section{El chacolí en la cultura popular}

Tanto en la poesía recogida en la Lira Popular como en aquella de tradición más letrada, encontramos algunas menciones al chacolí. En estos versos de Patricio Miranda Venegas, editor de La Lira Porteña, el chacolí forma parte del recuento de varias comidas y bebidas: "Quesitos de Putaendo,/los calabozos de ají,/ en casa del falte León,/ chicha, vino y chacolí (...) quién no tomaría así,/ aguardiente con anís/ a cuartillo la medida,/ y en las Coimas se vendía/ chicha, vino y chacoli". Resulta interesante, por cierto, el modo en que "chacolî" se utiliza al final de los versos para proponer llamativas combinaciones de una rima poco común, por ejemplo con “ajî”. José Hipólito Casas Cordero, en cambio, lo menciona como parte de una escena más trágica como consecuencia de una fiesta: "El crimen fué horrible i feo/ Como lo escribo en mi plana/ Chicha en una damajuana/ Tenian i chacolí/ Luego una muerte hubo allí/ Estando en una jarana".

El vínculo del chacolí con la vida cotidiana y la cultura popular se reflejó también en las campañas comerciales por medio de la prensa. En este contexto se produjo la difusión de "La Cueca del chacolí Rosado", publicada en El Mercurio el 21 de septiembre de 1914. El objetivo era promover las ventas de "El Patriarca de Noé", casa de comercio ubicada en Santiago, y apeló a este formato popular para llamar la atención de su clientela (Figura 1):

Un Chacolí rosado/ vende "El Patriarca"/ uno que no he probado/ en mi comarca. Ese Chacolí, ¡ay, sí!/ es de lo rico; / y solito me bebí/cántaro y pico.

Cántaro y pico, sí/ y más quisiera / ¡Si se parece a ti/ china hechicera!...

¡Como tú, rosadito/ también es él,/y semeja un traguito/ de pura miel!

Yo te tragara a ti/ tal como a él./ ¡Eres de Chacolí/ lindo tonel!

Un dieciocho tomé/ tanto "rosado" / que al tope me piqué. Quedé curado. 


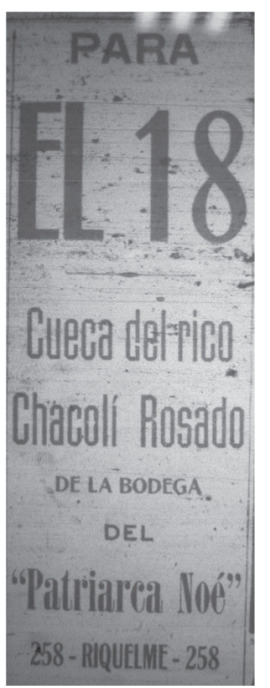

Figura 1. Promoción de " la Cueca del chacolí rosado" para motivar ventas de Bodega del Patriarca Noé (El Mercurio, 1914).

Y a la pampa, después/ en carretela/ rápido las eché/ con mi chicuela, sí

Y me saquearon/ y hasta el poncho a mi/ ¡Ah! Me robaron/ el Chacolí rosado.

Con mi chicuela/ con mi chicuela, sí/ y me saquearon/ y hasta el poncho a mi ¡Ah!, me robaron./ El Chacolí rosado/ nunca aflojó pues todo lo robado/ apareció. Él protege a sus clientes/ en todo caso/ todos son sus parientes./ ¡Es tan buenazo! Con Chacolí rosado/ este Dieciocho/ yo quedaré curado/ por siete u ocho.

Celebrando las glorias de O'Higgins, Carrera/ y todas las victorias/ que Dios nos diera.

Chacolí del "Patriarca"/ no tiene nada igual/ si tiene en sí la marca/ ¡Es celestial! ¡Celestial!/ ¡Ay! ¡Sí!/ Prenda del alma/ yo te compraré a ti/

por damajuana/ y pipas grandes/ Sí! Ay! Ay! Ay!

Esta "Cueca del Chacolí Rosado" refleja la mixtura de esta bebida con la cultura popular, como medio eficiente de celebración de las fiestas patrias del 18 de septiembre en Chile. El poema recorre el panteón de los padres fundadores de la Patria chilena, comenzando con Bernardo O'Higgins y José Miguel Carrera, enemigos en su tiempo, reconciliados en el alma chilena por medio de la celebración patria y el chacolí. Ambos personajes se enhebran en una historia con la mujer amada, con quien se unen, y con los ladrones, de quienes protegen. El chacolí tiene entonces tres funciones: une al hombre y a la mujer; une a los chilenos en su historia; y protege al pueblo del delincuente. Todo ello se asocia con la marca de la casa comercial "Patriarca Noé". El chacolí es la hebra mágica que logra todos estos objetivos.

Posteriormente Pablo de Rokha, en su Epopeya de comidas y bebidas de Chile, lo menciona varias veces, con naranjas, y siempre en cantidades copiosas: "la persona está sentada principalmente en un espino del Sur, quemado, pero con viento tremendo,/ no tomando, sino bañándose en el buen Chacolí de octubre, que gritará lleno de banderas" (21); "el causeo de patitas, que debe comerse en Codegua, no después de beber bastante Chacolí con naranjas amargas" (9); "el trago no bébalopuro, bébalo puro y con torrejas de naranja de la más agri-acida que encuentre, naturalmente en el naranjo más anciano de la aldea,/ báñese en Chacolí fuertón y corajudo" (14).

\section{El chacolí en la prensa}

Entre fines del siglo XIX y comienzos del XX, la prensa chilena dedicó un espacio considerable a la promoción del chacolí. Los avisos eran contratados por las empresas comerciales que se interesaban en comprar y vender chacolí. La colección de estos avisos constituye un rico corpus documental, a partir del cual se pueden inferir las prácticas culturales en torno a este producto.

En la década de 1870 el chacolí estuvo muy presente en los medios. Se publicaban avisos de compra y venta de esta bebida. El interés por ella se reflejaba en el espíritu de estos avisos. Un buen ejemplo fue el anuncio que se publicó reiteradas veces en enero de 1878 en El Ferrocarril. El texto señalaba "Vinos, chichas y Chacolíes, cualquiera que sea su estado, compran calle de Picarte $\mathrm{N}^{\circ} 4$ ". El lenguaje del anuncio denota cierta ansiedad por adquirir el producto. El aviso se publicó en forma reiterada los días 11,19 y 21 de enero de ese año (Figura 2).

Respecto de la materia prima, los avisos solían destacar las uvas con las que se elaboraba el chacolí. Un papel destacado tuvieron el Torontel, o Moscatel Amarillo (llamado Torrontés en Argentina). Se trata de una uva criolla, nacida del cruce entre Moscatel de Alejandría y Uva País. Un aviso ofreciendo 


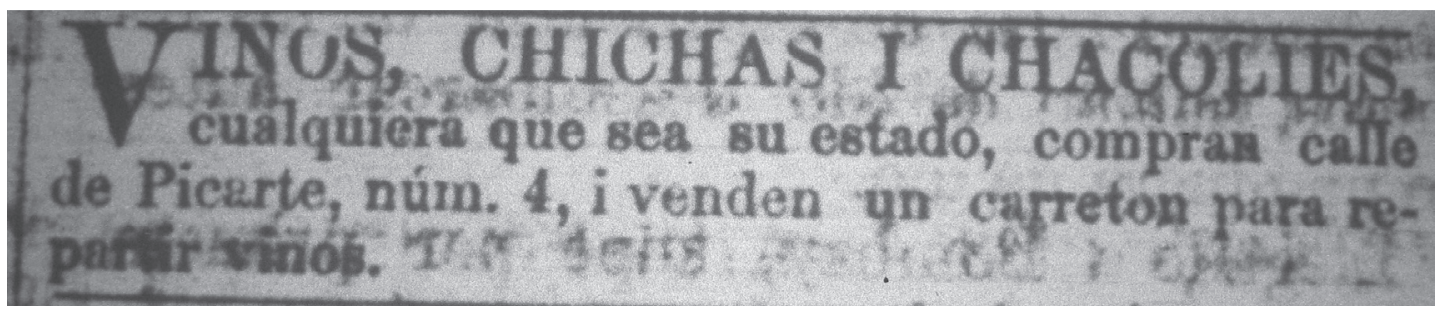

Figura 2. Anuncio Publicado repetidas veces en enero en El Ferrocarril (1878).

"Chacolí Torontel de pura uva" se publicó en $E l$ Mercurio el 9 de octubre de 1915. Poco antes, el 10 de agosto de 1915, en ese mismo medio otra casa demandaba la compra de chacolí blanco torontel. Posteriormente, el 16 de octubre de 1915, la empresa Ribas y Compañía demandaba la compra de 4.000 a 5.000 @ de chacolí torontel.

Otro aspecto al que poner atención son las referencias al lugar de origen como argumento de venta y valoración del producto. Esta fue una práctica ampliamente difundida en esa época. A veces, no era un lugar general, sino una hacienda particular, que trataba de fortalecerse como símbolo de garantía de calidad. Un buen ejemplo fue el chacolí de la Hacienda Almahue, que se vendía en el depósito que poseía en el centro de Santiago, en calle Chacabuco $\mathrm{N}^{\circ}$ 61. El aviso formulaba los argumentos de venta de este chacolí: "La pureza de este chacolí, su exquisito sabor y la modicidad de su precio, hacen de él una bebida indispensable y un elemento insustituible para las Fiestas Patrias" ( $E l$ Mercurio, 15 de septiembre de 1915). La Hacienda destacaba tres características centrales de su chacolí: pureza, exquisito sabor y bajo precio. Estos eran los argumentos para aspirar a ocupar un papel central en los días más importantes del año para los chilenos: las Fiestas Patrias de septiembre.

Respecto de los precios y envases, los avisos de prensa también entregan información relevante. El chacolí se vendía en pipas de madera o en damajuanas de vidrio. No se comercializaba en botellas. Los precios variaban según la cantidad. En cuestión de precios, también aparecen datos de interés. Como se ha señalado, en las décadas de 1830 y 1840, el chacolí se valuaba alrededor de ocho reales la arroba, según los registros notariales y las observaciones de Claudio Gay (1855). Después de la Guerra del Pacífico los precios fueron en aumento. Por ejemplo, en 1888, se ofrecían 1000 @ de chacolí blanco, a 18 reales por arroba en el Valle del Aconcagua (El Comercio de San Felipe,
7 de abril de 1888). La tendencia se profundizó en el siglo XX. En vísperas de la Primera Guerra Mundial, el chacolí rosado se vendía a \$ 0,50 el litro, $\$ 8,50$ la damajuana y $\$ 16,50$ la arroba $(E l$ Mercurio, 1 de octubre de 1914).

Los avisos comerciales publicados en la prensa de la época y conservados en las hemerotecas de las principales bibliotecas públicas de Chile, son un reflejo del período de auge del chacolí como producto típico chileno, el que se vio proyectado en los medios masivos de comunicación y en la vida cotidiana del país (Figura 3).

\section{Vinos típicos y cultura popular: la Fiesta del Chacolí}

Con el avance del siglo XX, los hábitos de consumo de vino en Chile tuvieron una tendencia a priorizar los vinos de tradición francesa y a dejar de lado los productos típicos tradicionales, entre ellos, el chacolí. Este producto perdió la posición hegemónica que tuvo en el tercer tercio del siglo XIX, cuando llegó a representar el 28\% de la producción vitivinícola nacional. La producción se retrajo, lo mismo que el consumo y la comercialización. La importancia relativa del chacolí experimentó un fuerte retroceso.

De todos modos, su valoración como producto típico se mantuvo presente en las zonas rurales del Valle Central, particularmente en las zonas más tradicionales. El ejemplo más representativo fue el municipio de Doñihue, localidad de 20.000 habitantes, ubicado en la provincia de Colchagua, en la VI Región. En 1975 esta comuna comenzó a celebrar la Fiesta del Chacolí, en la que se ponía en valor el conjunto de las tradiciones y productos artesanales y tradicionales de la región. Junto con el chacolí, en esta fiesta se exponían comidas típicas y tejidos de profundo arraigo regional, como los chamantos. También se incluyeron presentaciones artísticas de música popular. La iniciativa tuvo su 


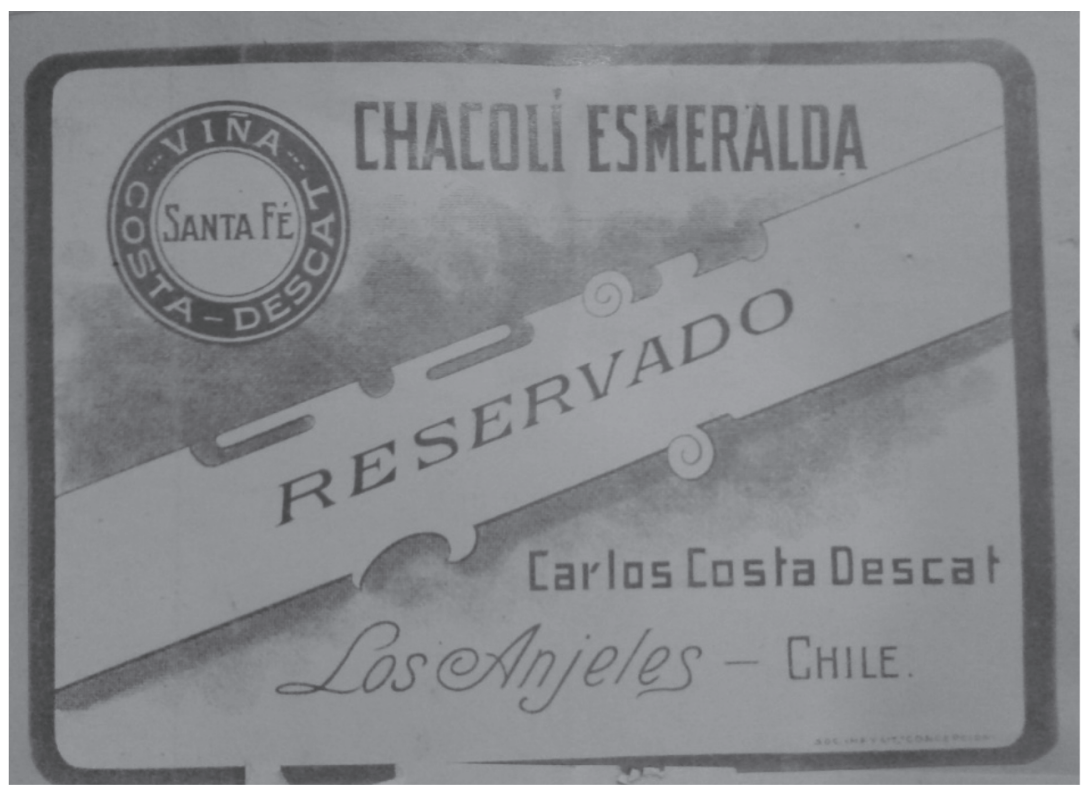

Figura 3. Etiqueta de chacolí registrada en el Instituto Nacional de la Propiedad Industrial el 26 de septiembre de 1922, por Carlos Costa Descat, productor de vinos y chacolíes en la localidad de Los Ángeles (500 km al sur de Santiago de Chile). Fuente: Archivo del INAPI.

continuidad y se ha mantenido vigente durante 40 años, como homenaje y reconocimiento a un vino típico chileno, que a pesar de no tener actualmente el peso comercial de otros tiempos, sigue vivo en la tradición de Chile profundo.

\section{Conclusiones}

El chacolí fue un vino liviano y popular, elaborado en Chile en los siglos XIX y XX, a partir de uvas criollas y de profundo arraigo popular. El Chacolí emergió como un vino típico, dentro del proceso general que se ha estudiado para el caso europeo (Coello, 2008). En los últimos años, el chacolí ha sido revalorizado en el País Vasco (Hidalgo, Buruaga y Ocete, 2012). En Chile ha tenido también su propio itinerario histórico.

El chacolí se hizo visible en las viñas chilenas desde comienzos del siglo XIX. Los registros más antiguos corresponden al valle del Huasco, en 1810. Poco después se extendió también al Valle Central. En los años siguientes experimentó un fuerte ascenso, hasta ocupar un lugar central en la industria vitivinícola nacional. En su momento de apogeo, durante la segunda mitad del siglo XIX, se elaboraban 16 millones de litros anuales de chacolí, lo que representaba un tercio de la industria vitivinícola chilena. Dentro de este ciclo de auge, el chacolí se extendió hacia la vida económica, social y cultural del país. Inspiró poemas y canciones populares, particularmente, cuecas, lo que muestra el profundo arraigo cultural que alcanzó en el alma chilena.

El auge del chacolí se extendió desde el siglo XIX hasta comienzos del XX, cuando comenzó a decaer. En el siglo XX se produjo su desplazamiento por los vinos de uvas francesas. Duramente criticado por los tecnócratas europeos, el chacolí fue estigmatizado y marginado por los mercados. Los consumidores lo abandonaron masivamente, y quedó reducido a un pequeño segmento del mercado, con bajo poder adquisitivo y concentrado en las zonas rurales. De todos modos, todavía se nota la persistencia del chacolí en espacios muy específicos. Su principal referencia se visualiza en la Fiesta del Chacolí, celebrada por el Municipio de Doñihue desde 1975 hasta la actualidad. Esta celebración es considerada parte del patrimonio intangible de Chile, y sirve de catalizador para valorar también la música popular con sus artistas, la gastronomía típica y las artesanías (en particular los chamantos).

Desde el punto de vista del mercado, la relevancia actual del chacolí es marginal en Chile. De todos modos, su tradición de más de doscientos años, su profundo arraigo en el alma del pueblo y su carácter de producto típico, lo sitúan en un lugar relevante 
como parte del patrimonio inmaterial de la nación, y esto representa una base considerable para su futuro desarrollo. El chacolí tiene un potencial digno de tener en cuenta.

\section{Agradecimiento}

Los autores agradecen al proyecto Fondecyt 1130096.

\section{Literatura Citada}

Alessandri, J.P.

1885. Fundo "San Pedro", cerca del lugar llamado Romeral, situado a $12 \mathrm{~km}$, al Este de la ciudad de Curicó, capital de la provincia del mismo nombre. Memoria presentada para obtener el título de Ingeniero Agrícola. Santiago, Instituto Agrícola.

Aránguiz Donoso, H.; Rodríguez Salas, C.

1996. Tradicionalismo y cambio agrícola en Aconcagua: elementos para su comprensión. Historia, 29: 29-33.

Briones, F.

2006. Lo inmigrantes franceses y la viticultura en Chile: el caso de René F. Le Feuvre. Universum, 21 (2): 126-136.

Charlín Recabarren, J.

1881. Memoria sobre el fundo Porvenir de Huechagüe, perteneciente a los señores Espinoza y Fernández, sito en el Departamento de Cauquenes. Presentada para optar al

Coello, C. título de Ingeniero Agrícola. Santiago, Instituto Agrícola.

2008. El Sistema Jurídico de las Denominaciones de Origen. Las Bases Históricas y Administrativas del Derecho Vitivinícola Español. Sevilla, Instituto Andaluz de Administración Pública, 1460 pp.

Del Pozo, J.

2014. Historia del vino chileno. Desde la época colonial hasta hoy. Santiago, LOM Ediciones, 306 pp.

Fernández Labbé, M.

2010. Bebidas alcohólicas en Chile. Una historia económica de su fomento y expansión 1870-1930. Santiago, Centro Barros Arana/Universidad Alberto Hurtado, 270 pp.

Fernández Niño, P.

1817. Cartilla de campo y otras curiosidades, dirigidas a la enseñanza y buen éxito de un hijo. Santiago, 445 pp.

Fernández Niño, P.

1867. Cartilla de campo. Escrita para el uso de los agricultores. Segunda edición aumentada y corregida. Santiago, Imprenta del Independiente, $118 \mathrm{pp}$.

Gay, C.

1855. Historia física y política de Chile. Tomo II: Agricultura. Santiago, Museo de Historia Natural, 1855. Edición moderna consultada: Santiago, Icira, 1973, 443 pp.

Hanisch, W.

1976. El arte de cocinar de Juan Ignacio Molina. Chile, Ediciones Nihil Mihi, $150 \mathrm{pp}$.

Hidalgo, J.; Sáenz de Buruaga, T.; Ocete, R.

2012. Vid cultivadas y silvestre en el territorio de la antigua diócesis de Valpuesta (Álava, Burgos y Cantabria, España): un acercamiento a la historia del vino Chacolí. Revista Estudios Avanzados, 18: 101-130.

Lacoste, P; Briones, F.; Jiménez, D.; Rendón, B.

2014. La Denominación de Origen Pisco en Chile: algunos problemas nacionales e internacionale. Idesia, 32 (2): 47-56.
Lacoste, P.; Jiménez, D.; Castro, A.; Rendón, B.; Soto, N. 2013. A binational Appellation of Origin: Pisco in Chile and Peru. Chilean Journal of Agricultural Research, 73 (4): 107-114.

Le Feuvre, R.; Barros, L.; Ovalle, D.; Rojas, M.

1886. Exposición Departamental de Los Andes. Informe de la Comisión nombrada para estudiar dicha exposición. Boletín de la Sociedad Nacional de Agricultura, vol. XVIII, 5 de noviembre de 1886.

Le Feuvre, R.

1890. Informe pasado al señor Ministro de Industria y Obras Públicas sobre el estado actual de la agricultura y muy especialmente de la viticultura en la provincia de Maule y los medios de fomentar estas industrias. Boletín de la Sociedad Nacional de Agricultura, vol. XXI, 20 de julio de 1890.

Le Feuvre, R.

1884. Informe sobre el estado de las viñas en San Felipe, Los Andes, Mendoza, San Juan y Buenos Aires. Boletín de la Sociedad Nacional de Agricultura, vol. XV, 5 de junio de 1884.

Le Feuvre, R.

1877. Informe sobre la enfermedad de las viñas presentado al Ministro de Hacienda. Boletín de la Sociedad Nacional de Agricultura, vol. VIII, 20 de marzo de 1877.

Menadier, J.

1874. La vinicultura nacional. Boletín de la Sociedad Nacional de Agricultura, vol. VI, 5 de noviembre de 1874.

Morales, J.

1896. Historia del Huasco. La Serena, Universidad de Chile, 1981, 348 pp.

Pereira Salas, E.

2013. Apuntes para la historia de la cocina chilena. Santiago, Uqbar, 100 pp.

Pszczólkowski, P.

2014. "Terroir" y "Climats": ¿realidad o quimera? Revista Iberoamericana de Viticultura, Agroindustria y Ruralidad (RIVAR), 1 (1): 13-19.

Pszczólkowski, Ph.

2015. Sauvignon Blanc, Cabernet-Sauvignon y Carmenère, cepas claves de la viticultura actual de Chile. Revista Iberoamericana de Viticultura, Agroindustria y Ruralidad (RIVAR), 2 (1): 1-16.

Pszczólkowski, Ph.

2013. Carmenere, mayoría de edad: 1994-2012. Estudios Avanzados, 20: 137-154.

Riveros, V.

1881. Memoria sobre el fundo de Quella perteneciente a la testamentaria del finado señor Juan Manuel Oñat. Presentada al Instituto Agrícola para optar al título de Ingeniero Agrícola. Santiago, 1881, 40 pp. 


\section{Notas}

1 Tasación de bienes de don Francisco Prats, Santiago, 10 de julio de 1837. AN, Fondo Judiciales de Santiago, Legajo 177, pieza 4, foja $1 \mathrm{v}$.

2 Don Bernardo González con don Mateo Campos sobre cobre de pesos, Santiago, 1844. AN, Fondo Judiciales de Santiago, Pieza 9, foja 18v.

3 Anulación de Venta de tres cubas de chacolí, Santiago de Chile, 11 de julio de 1845. AN, Fondo Judiciales de Santiago, Legajo 35, Pieza 7, tomo 1.
4 Cesión de bienes a acreedores por parte de Ceciliano Álvarez, Santiago, 13 de junio de 1845. AN, Fondo Judiciales de Santiago, legajo 35 , pieza 1 , foja $3 \mathrm{v}$.

5 Inventario y tasación de bienes de don Joseph Galdame, San Felipe, 26 de junio de 1834. AN, Fondo Judiciales de San Felipe, Legajo 24, Pieza 7, foja 23v.

6 Partición de bienes de don Pedro Figueroa, San Felipe, 25 de julio de 1844. AN, Fondo Judiciales de San Felipe, Legajo 21, Pieza 20, Foja 6v. 